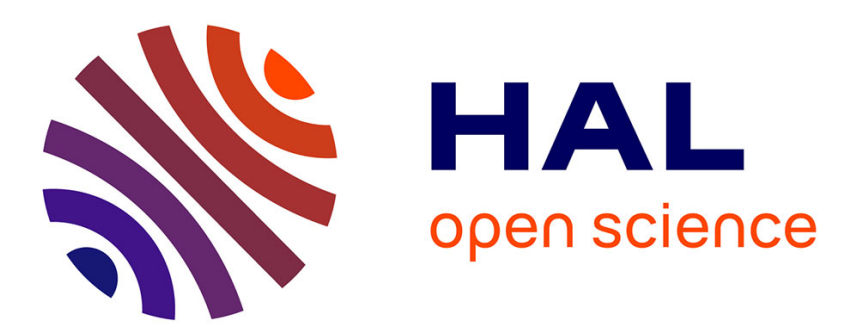

\title{
Transmission System Operator Regulation for Electric Vehicle Fleets: A Survey of the Issues
}

\author{
Yannick Perez, Marc Petit
}

\section{To cite this version:}

Yannick Perez, Marc Petit. Transmission System Operator Regulation for Electric Vehicle Fleets: A Survey of the Issues. The Automobile Revolution, Springer, pp.135 - 146, 2016, 978-3-319-45837-3. 10.1007/978-3-319-45838-0_9 . hal-01424647

\section{HAL Id: hal-01424647 https://hal.science/hal-01424647}

Submitted on 2 Jan 2017

HAL is a multi-disciplinary open access archive for the deposit and dissemination of scientific research documents, whether they are published or not. The documents may come from teaching and research institutions in France or abroad, or from public or private research centers.
L'archive ouverte pluridisciplinaire HAL, est destinée au dépôt et à la diffusion de documents scientifiques de niveau recherche, publiés ou non, émanant des établissements d'enseignement et de recherche français ou étrangers, des laboratoires publics ou privés. 


\section{Transmission System Operator Regulation for Electric Vehicle Fleets: A Survey of the Issues}

Yannick Perez 1,*

Phone +33631618728

Email yannick.perez@u-psud.fr

Marc Petit 2

Email marc.petit@supelec.fr

RITM Lab and LGI CentraleSupélec, 91190 Gif-sur-Yvette, France

2 GeePs, CNRS UMR 8507 CentraleSupélec, UPSud and UPMC, 91190 Gif-sur-Yvette, France

\section{Abstract}

A modular framework is used to analyze how Grid Integrated Vehicles (GIVs), i.e. bi-directional plug-in electric vehicles that are able to modulate their charging rate and have bi-directional capabilities, could be managed efficiently to deliver grid services for transmission operators and conversely, how these new services could be set aside by the design of the current rules in some regions. Based on a detailed analysis of the rules implemented by some representative TSOs, we discern two modules that gather the essential rules for GIV development: the rules towards aggregation of EVs, and the rules defining the payment scheme of the services provided by GIVs. We deduce an optimal combination among these rules that could define the ideal organization for GIVs. Finally, we confront this ideal TSO organization with the European guidelines under construction.

AQ1

$\mathrm{AQ} 2$

\section{Keywords}

Electric vehicles

Transmission system operator

Market design

\subsection{Introduction}

It is puzzling today to analyze the organizational diversity of Transmission System Operators (TSOs) in competitive electricity markets around the world (Rious et al. 2008). In liberalized power systems, TSOs present different organizational forms or governance structures, depending on network ownership, on applied regulation, etc. In order to understand this wide range of organizational forms, we argue that the use of Wilson (2002) and Baldwin and Clark (2002) modular frame is helpful in defining and classifying the rules of the game from the most to the least adapted to new services and innovations.

Considering research dealing with Grid Integrated Vehicles (GIVs), i.e. plugin electric vehicles that are able to modulate their charging rate and have bidirectional capabilities, coalitions of electric vehicles (EVs) are likely to be integrated into TSO reserves in the future (Han et al. 2010; Kempton and Letendre 1997; Kempton and Tomić 2005a). In this respect, GIVs will become Reserve Providing Units, which are required and financed by the TSO set of rules that are today very differently organized around the world.

In this paper, we apply the aforementioned modular frame to analyze how GIVs could be managed efficiently to deliver valuable services for transmission operators and conversely, how these new services could be set aside by the design of the current rules in some regions. 
This work builds on (Codani et al. 2014) which already identified the representative TSO rules for GIVs providing frequency control. In this paper, the authors make a comparison among five representative TSOs on a list of rules and characteristics that are important for GIV deployment. The five TSOs in question, and the regulation manuals associated are: Energinet.dk (2012), RTE (2004, 2011a, b), ERCOT (2012, 2013a, b , c , d), CAISO (2010, 2011, 2013) and PJM (2012, 2013a , b , c). The National Grid Company of the UK has been added in this paper to increase the diversity of possible rules regarding demand side response (DSR) regulation and remuneration (National Grid 2012a, b, 2013). The term DSR encompasses bidirectional demand services, which will enable to take full advantage of EV potential.

Based on this previous literature, we go a step further and identify two key modules that gather the essential rules for GIV development: the rules towards aggregation of EVs, and the rules defining the payment scheme of the services provided by GIVs. The novelty in this work relies on a deeper and more policy-oriented analysis of the most important rules detailed in Codani et al. (2014). Moreover, we analyze the proposed ENTSOE network codes, in order to shed some light on the possibility of implementing the ideal TSO in reality.

The paper is organized as follows. Sections 9.2 and 9.3 are dedicated respectively to the identification of the best aggregation rules and payment schemes for grid services. In Sect. 9.4, we propose an ideal TSO organization, and compare it to the 6 representative TSOs understudy. In Sect. 9.5 we discuss some policy recommendations by screening European issues and ENTSOE network codes through the lenses of our framework. Last section concludes our main findings.

\subsection{Module 1: Aggregation Rules}

An aggregator has a fundamental role in GIV architectures for TSO services: it is responsible for presenting a fleet of EVs as a single entity to the TSOs. Aggregators are required because: (a) TSOs deal with large entities (MW rather than kW size), (b) TSO data processing capabilities do not have the bandwidth for controlling millions of $\mathrm{kW}$ size units; they were designed for 100s of multi-MW sized units, and (c) TSOs expect their resources to be reliable, which is a problem for a single EV. An EV necessarily gives first priority to transportation, but from the power system perspective one EV may leave the power system at any moment. Aggregators can address these issues by controlling a large number of EVs (Kamboj et al. 2011; Kempton and Tomić 2005b), and offering a single, statistically-reliable entity to the TSO. Finally, aggregators should also be able to deal with a large diversity of degrees of information and degrees of uncertainty induced by many different vehicle types, driver plans, and regularities in driver behaviors (Kempton and Letendre 1997; Bessa and Matos 2010), details well outside the business expertise or interest of TSOs.

Correspondingly, TSOs must allow such aggregation for GIV use, but what are the main rules to do so? Here we would like to insist on three rules: the size of the minimum bid, the interoperability among DSOs, and the technical form of aggregation.

\subsubsection{The Size of the Bid}

In all reserve markets, bids cannot be less than a minimum power level; we have seen a range of minima from $100 \mathrm{~kW}$ (PJM, frequency regulation) to $10 \mathrm{MW}$. In terms of EV coalitions, this minimum-bidding amount can be converted into a minimum number of EVs. A high level of minimum bidding amount would represent a challenge for the development of pilot and early commercial projects, because they may not have enough vehicles to meet with the minimum.

For instance, considering charging stations of $3 \mathrm{~kW}$ (domestic plugs), and that one EV out of three is available for reserve markets (because of transportation, charging needs), the minimum fleet size would be 100 vehicles for a minimum bid value of $100 \mathrm{~kW}$. On the other side, given a minimum bidding size of $10 \mathrm{MW}$, the number of EVs in a coalition should be at least 10,000. These figures should be put in perspective with those of today EV sales; in 2013, only around 10,000 EVs were sold in France for example. 
Thus, given a high minimum bidding size, it would be impossible to make a coalition of privately owned vehicles in France, not to mention a company fleet.

Even if we consider a high penetration of EVs, say, in 10 years, a high minimum bidding value would narrow the diversity of potential aggregators: among others, company fleets of utility vehicles, or Vehicle-toBuilding scenarios (fleets gathering vehicles parked in the same parking lot), would not be allowed to become aggregators.

\subsubsection{The Scope of the Bids}

Single or multiple DSO zones of technical regulation are a second key concern for aggregation business. As EVs are small moving storage entities, TSOs rules should also allow resources that may shift locations, and may be spread across electrical distribution companies (EDCs). One way to manage that may be to register charging stations rather than registering EVs. Regarding movement across EDCs, some TSOs work with very few EDCs (for instance RTE, whose main EDC partner is ERDF) but others are connected to many of them (for instance Energinet.dk, with $65 \mathrm{EDCs}$ ), and in the latter, more typical case, not being allowed to aggregate across EDCs makes aggregation more challenging or even impossible.

Thus for our modular frame, the best option is to allow and organize interoperability among various DSOs as done in RTE or Energinet.dk. From an aggregator point of view, a restrictive implementation of this rule could be very constraining. Indeed, the minimum fleet size is induced by the rationale described in Sect. 9.2.1, and if this minimum has to be reached in a single DSO area, it may be impossible for aggregators to meet with the minimum fleet size requirements.

\subsubsection{The Precision of the Action}

Our last criterion is a distinction between telemetry and financial aggregations. Telemetry is the desired form of aggregation; it enables combining bids and then controlling distributed power flows from one or more central locations. In contrast, financial aggregation only allows combining financial bids but not power flows, which, among other things, would prevent an aggregator from implementing dispatching algorithms.

In our frame of combining the best rule issued from the TSOs survey, we propose to select the PJM and Energinet.dk rules that allow the telemetry aggregation. It is noticeable that TSOs may be unwilling to allow large telemetry aggregations, because it would make verification of reserve activation more difficult.

\subsubsection{Partial Conclusion}

The Table 9.1 sums up the identified rules regarding aggregation and the different possible organizations for each rule.

\section{Table 9.1}

The different organizations for Module 1

\begin{tabular}{|c|c|c|}
\hline \multirow{2}{*}{ Aggregation rule module } & \multicolumn{2}{|c|}{ Organization } \\
\hline & Best option & Restrictive option \\
\hline Size of the bid & $100 \mathrm{~kW}$ & $10 \mathrm{MW}$ \\
\hline Interoperability among DSOs & Possible & Impossible \\
\hline Aggregation level & Telemetry & Financial \\
\hline
\end{tabular}




\subsection{Module 2: The Rules Defining the Payment Scheme of Grid Services}

\subsubsection{Regulated of Market Based?}

For a given reserve market, TSOs may differ in their way of dispatching the required power among all the units that are part of the reserve in question (whether primary or secondary). Raineri et al. (2006) identified several transaction mechanisms. Some TSOs implement open markets in which units are allowed to bid as they want in these markets, a bid being an amount of offered capacity and its associated price. Bids are either accepted or rejected by the TSO. Other TSOs dispatch the total required power among all the units that are part of the reserve in proportion to their historical load share. In this situation, depending on the TSOs, providing reserve is either a choice or compulsory for a unit.

As a partial conclusion in our framework, the use of market prices as a way to determine the dispatch of reserves is much more profitable for new storage resources such as GIVs. First, in a regulated approach, we would have to wait for a formal change of the rules, so that they would be suitable for new resources such as GIVs. This adaptation is likely to be lengthy, sub-optimal, and lagging EV sales, trying to catch up with the market evolution instead of taking the lead. Then, changing a contract binding an aggregator and the TSO would also be a lengthy process, one that is not really compatible with a dynamic EV fleet. Indeed, in addition to many dynamic changes within a fleet because of transportation needs, the EV fleet itself is also likely to evolve, with new EVs joining or leaving the program.

\subsubsection{Complete or Incomplete?}

Besides the nature of the payment scheme, the second element of our frame is its consistency regarding the services offered or possibly offered by EVs. It is puzzling to identify ancillary services that are required but not remunerated specifically by some TSOs and DSOs. Some services are just mandatory with no explicit remuneration nor explicit reserve allocation method. Examples are for instance PJM or CAISO not paying for primary frequency regulation.

Regarding our analysis, the more the payment scheme is incomplete and does not compensate for the services provided by all the actors, the more EVs are penalized in their contribution as GIV resources. A clear and complete payment scheme is needed as a condition in the Ideal TSO we seek to build.

From the TSO perspective, it could be beneficial to complete the payment scheme of ancillary services. Indeed, as AS providing units do not have any incentive to provide these unpaid services, they sometimes achieve poor performance in the provision of these services. For instance, Ingleson et al. (2009) point out the fact that the total frequency droop (also referred to as frequency characteristic) of Eastern Interconnection in the US has been dangerously decreasing for the past 10 years, jeopardizing grid security. AQ3

\subsubsection{Additional Bonus for Intense Flexibility Providers?}

In the United States, the Federal Energy Regulatory Commission (FERC), a federal agency responsible for harmonizing interstate energy laws and TSO rules, has investigated the different frequency regulation compensation practices of TSOs in (Federal Energy Regulatory Commission 2011). Its conclusion is that current compensation methods are unjust and discriminatory, specifically because fast ramping resources (resources that are able to change their output very quickly) are not remunerated enough with respect to the greater amount of frequency regulation provided.

To deal with this issue, the FERC makes two recommendations. First, remuneration should not only be based on availability (i.e. in $\$ / \mathrm{MW}$ ), but also on utilization $(\$ / \mathrm{MWh})$, and every MWh exchanged with the grid for the purpose of frequency control should be counted as a source of positive revenue for the resource in question, whether the MWh flowed from the grid to the resource or from the resource to the grid. That way, as fast-ramping resources respond faster, they exchange more MWh with the grid than slow-ramping units, so payment will be fairer. 
Second, regulation resources should receive a two-part payment: the first one is the capacity and utilization payment discussed above, including an opportunity cost, and the second one is based on performance, taking into account the response accuracy. Further details about the performance calculation are provided in the more recent FERC order 784 (Federal Energy Regulatory Commission 2013a) speed and accuracy should be taken into account in the payment of ancillary services.

EVs are very fast-ramping resources. Therefore, TSOs that abide by FERC compensation recommendations, or similar compensation schemes reflecting the value of fast responses, are more attractive for GIV aggregators. Considering our optimal TSO, the best solution is then to be able to benefit from this kind of bonus. However, the implementation of this bonus should be managed carefully. Indeed, the addition of an extra bonus to an existing payment scheme should be set at the efficient level. The risk induced by introducing a bonus is that it might create a distortion that could either overcompensate the initial problem, or not compensate enough and leave the issue unsolved.

An alternative way of proceeding would be to regard fast and slow ramping tenders as two different products Thus, establishing a separate market earmarked for fast-ramping resources, with its own rules and regulations, might be another solution to remunerate these services in a just and fair manner. At last, some electrical grids might not presently feel the need for fast-ramping resources. The droop control method of conventional units has been operating for a long time and seems to be working quite well (provided that financial incentives are adequate, see Sect. 9.3.2). Thus, introducing faster responses in this context has to be investigated thoroughly by the TSOs. In a first time, such services may be mostly suited to extreme frequency containment plans after severe disturbances rather than for normal operations. Then, with the increasing penetration of intermittent renewable sources, which induce more production fluctuation and less system inertia, fast-ramping units may be more and more required. We are already observing this phenomenon in some island networks, which are isolated - so very sensitive to frequency drops - but benefit from substantial wind and solar resources.

\subsubsection{Partial Conclusion}

The Table 9.2 sums up the rules dealing with the payment scheme, and the different possible organizations.

Table 9.2

The different organizations for Module 2

\begin{tabular}{|l|l|l|}
\hline \multirow{2}{*}{ Payment scheme module } & Organization & \multicolumn{2}{l}{} \\
\cline { 2 - 3 } & Best option & Restrictive option \\
\hline Nature of the payment & Market based & Incomplete scheme \\
\hline Form of the payment & Complete scheme & Not existing \\
\hline Bonus for flexibility & Set at the efficient level, or separate market created & Noted
\end{tabular}

\subsection{The Ideal TSO for Grid Integrated Vehicles}

The optimal implementation of the TSO rules for GIVs providing Demand Side Response, resulting from a combination of the organizations previously presented, is displayed in Table 9.3. Within this ideal TSO, there is almost no barrier to the building of EV coalitions. The latters can be part of all reserves, which are all remunerated in a fair way. Therefore in this TSO, small company fleets are aggregated and participate into reserve markets. Similarly, privately owned parking open to public (in malls, or commercial buildings) can aggregate their charging stations and offer their customers to provide grid services. These two kinds of fleets could provide local DSO services, such as load shifting or voltage regulation, as well as TSO services. Then, with the increasing EV penetration, larger fleets, participating to widespread TSO services spread over 
multiple DSO areas, would be aggregated. They could take advantage of being very statistically reliable, though still very efficient.

Table 9.3

Ideal TSO organization

\begin{tabular}{|l|l|}
\hline Rule & Best organization \\
\hline R1: Size of the bid & $100 \mathrm{~kW}$ \\
\hline R2: Scope (multiple DSO) & Possible \\
\hline R3: Aggregation level & Telemetry \\
\hline R4: Nature of the payment & Market based \\
\hline R5: Consistency of the payment & All AS services should be paid \\
\hline R6: Bonus for extra flexibility & Set at the efficient level, or separate market created \\
\hline
\end{tabular}

It is noticeable that this table results from an analysis of representative TSOs. We cannot logically exclude the fact that some non-studied TSOs may enjoy even more favorable rules that would be missing in our ideal TSO. However, we chose a wide diversity of TSOs, including some at the forehead in terms of regulation for new technologies and smart grids. Thus, despite the fact that we may have missed some particular rules, we would like to show that using this comparative frame already deliver some valuable insights.

In order to do so, we compare the TSOs understudy with the ideal one. Results are presented in Table 9.4.

\begin{tabular}{|c|c|c|c|c|c|c|}
\hline TSO & R1 & R2 & R3 & R4 & R5 & R6 \\
\hline RTE & $\mathrm{x}$ & 7 & $r$ & $\mathrm{x}$ & 7 & $x$ \\
\hline PJM & $r$ & $\mathrm{x}$ & $r$ & $r$ & $\mathrm{x}$ & $r$ \\
\hline ERCOT & $r$ & $\mathrm{x}$ & $x$ & $r$ & $\mathrm{x}$ & $\mathrm{x}$ \\
\hline Energinet.dk & $\sim$ & $r$ & $r$ & $r$ & $r$ & $\mathrm{x}$ \\
\hline CAISO & $\sim$ & $\mathrm{x}$ & $x$ & $r$ & $x$ & $r$ \\
\hline NGC & $x$ & $r$ & $r$ & $\sim$ & $x$ & $x$ \\
\hline
\end{tabular}

\section{Table 9.4}

Evaluation of

the

representative

TSOs

We can infer two main conclusions from this table. First, there is no TSO implementing a perfect regulation favorable to the development of GIV. However, some of them are closer to the ideal one than others. Second, our frame can be used as a methodological tool, which can be applied to other TSOs in order to assess their friendliness towards GIV deployment, and guide reforms towards what should be done to go a step further.

\subsection{Policy Recommendations Towards ENTSOE}

In this part, we try to see whether the ideal TSO as described in Sect. 9.4 is reachable with ENTSOE recommendation, which are provided in the Network Codes. The latter's are still under revision, but recent versions are publicly available. They should come into force in the following years, guiding TSOs in their organizations. 
The new ENTSOE network codes introduce the concept of Significant Grid User (SGU) (ENTSOE 2013a). The latter could be either a demand facility connected to the DSO, or an aggregator as defined in ENTSOE (2012), that is, a legal entity responsible for the operation of a number of demand facilities by means of demand aggregation. SGUs should be allowed to provide Demand Side Response (DSR) directly to the TSO, or via the DSO. (ENTSOE 2012) also suggests the implementation of DSR Very Fast Active Power Control (DSR VFAPC), defined as a very fast (within $2 \mathrm{~s}$ ) demand modulation in response to frequency fluctuations.

Moreover, TSOs should avoid undue barriers for new entrants, and make the participation to DSR for storage units easier (ENTSOE 2013b). With respect to the ENTSOE role model (ENTSOE 2011), it means that aggregators and energy storage units should be allowed to become Balance Responsible Parties (BRPs).

According to ENTSOE guidelines, units providing Frequency Containment Reserves (FCR) (also referred to as frequency response or primary control) should have the right to perform telemetry aggregation if the coalition size does not exceed 1.5 MW (ENTSOE 2013c).

The ENTSOE network code on Electricity Balancing (2013b) specifies that the procurement of Balancing Services should be market based and market based only.

Some rules are not addressed within the network codes because they are left at the discretion of each TSO. We can see that there is a good correlation between the ENTSOE guidelines and the ideal TSO; the network codes are paving the way for the implementation of a complete DSR framework suitable for all new controllable loads.

Table 9.5 makes a comparison between our ideal TSO and the ENTSOE future rules and guidelines.

Table 9.5

Ideal TSO versus ENTSOE guidelines

\begin{tabular}{|l|l|l|}
\hline Rule & Ideal TSO & ENTSOE proposals \\
\hline Minimum size & $100 \mathrm{~kW}$ & Not addressed \\
\hline $\begin{array}{l}\text { Interoperability } \\
\text { among DSOs }\end{array}$ & Possible & $\begin{array}{l}\text { Not clearly defined, but TSOs and DSOs should make all endeavors } \\
\text { and cooperate in order to ease the participation to DSR }\end{array}$ \\
\hline $\begin{array}{l}\text { Aggregation } \\
\text { level }\end{array}$ & Telemetry & $\begin{array}{l}\text { Status of aggregator defined. Telemetry aggregation considered for } \\
\text { FCR up to 1.5 MW }\end{array}$ \\
\hline $\begin{array}{l}\text { Nature of the } \\
\text { payment }\end{array}$ & Market Based & Market based \\
\hline $\begin{array}{l}\text { Incompleteness } \\
\text { of the payment }\end{array}$ & All AS should be paid & All AS should be paid \\
\hline $\begin{array}{l}\text { Extra bonus for } \\
\text { flexibility }\end{array}$ & $\begin{array}{l}\text { Set at the efficient } \\
\text { level/separate market } \\
\text { created }\end{array}$ & DSR VFAPC should be implemented \\
\hline
\end{tabular}

\subsection{Conclusion and Future Work}

In this paper, we used a modular frame, composed of two different modules, to define an ideal TSO for GIVs providing Demand Side Response. The rules of this ideal TSO would encourage the formation of EV coalitions, no matter neither their sizes nor their geographical expansions. All TSO services would be remunerated in a fair and just manner. The comparison between some representative TSOs and the ideal one reveal that some of them are not far from implementing the optimal combination of rules. The guidelines provided by ENTSOE network codes should strengthen this trend as the analysis of these documents outline their correlations with the ideal TSO. 
Our modular frame is of course not exhaustive. For instance, we did not deal with the status of energy storage units, which is not properly defined by each TSO (Codani et al. 2014) [although the FERC is pushing forward for the integration of these new units (Federal Energy Regulatory Commission 2013b)]. Further work could then consist in completing this study framework.

\section{Acknowledgments}

The authors gratefully acknowledge the contributions of Bjoern Christensen (Nuvve), Ole Jan Olesen and Yannick Phulpin (EDF R\&D).

This research benefits from the support of the Chair "PSA Peugeot Citroen Automobile: Hybrid technologies and Economy of Electromobility", so-called Armand Peugeot Chair led by Ecole Centrale Paris, ESSEC and SUPELEC and sponsored by PEUGEOT CITROEN Automobile.

\section{References}

C. Y. Baldwin and K. B. Clark, 2002. "The Option Value of Modularity in Design: An Example from Design Rules, Volume 1: The Power of Modularity," SSRN Electronic Journal, [Online]. Available:

http://www.ssrn.com/abstract=312404 .

R. J. Bessa and M. A. Matos, 2010. "The role of an Aggregator Agent for EV in the Electricity Market," in 7th Mediterranean Conference and Exhibition on Power Generation, Transmission, Distribution and Energy Conversion, no. November. IET, p. 9.

CAISO, 2013. “Tariff Clarifications to Implement Pay for Performance Regulation”.

CAISO, 2011. "Non-Generator Resources - Regulation Energy Management - Frequently Asked Questions". CAISO, 2010. "Proposal for participation of non-generator resources in California ISO ancillary services markets".

P. Codani, K. Willett, and L. Andrew, 2014. "Critical rules of transmission system operators for grid integrated vehicles providing frequency control," working paper.

Energinet.dk, 2012. Ancillary services to be delivered in Denmark Tender conditions.

ENTSOE, 2011. The Harmonised Electricity Market Role Model.

ENTSOE, 2012. Network Code on Demand Connection, [Online]. Available: https://www.entsoe.eu/majorprojects/network-code-development/demand-connection/

ENTSOE, 2013a. Network Code on Operational Security, [Online]. Available: https://www.entsoe.eu/majorprojects/network-code-development/operational-security/

ENTSOE, 2013b. Network Code on Electricity Balancing, [Online]. Available: https://www.entsoe.eu/majorprojects/network-code-development/electricity-balancing/

ENTSOE, 2013c. Network Code on Load-Frequency Control and Reserves, [Online]. Available: https://www.entsoe.eu/major-projects/network-code-development/load-frequency-control-reserves/

ERCOT, 2012. Nodal Protocols Section 8 : Performance Monitoring.

ERCOT, 2013a. Nodal Protocols Section 6 : Adjustment Period and Real-Time Operations.

ERCOT, 2013b. Nodal Protocols Section 4 : Day-Ahead Operations. 
ERCOT, 2013c. Nodal Protocols Section 3 : Management Activities for the ERCOT System.

ERCOT, 2013d. Nodal Protocols Section 16 : Registration and Qualification of Market Participants.

Federal Energy Regulatory Commission, 2011. "Order 755 - Frequency Regulation Compensation in the Organized Wholesale Power Markets".

Federal Energy Regulatory Commission, 2013a. "Order 784 - Third Party Provision of Ancillary Services; Accounting and Financial Reporting for New Electric Storage Techno”.

Federal Energy Regulatory Commission, 2013b. “Order 792 - small generator interconnection agreements and procedures".

S. Han, S. Member, S. Han, and K. Sezaki, 2010. "Development of an Optimal Vehicle-to-Grid Aggregator for Frequency Regulation," IEEE Transactions on Smart Grid, vol. 1, no. 1, pp. 65-72.

J. W. Ingleson, S. Member, E. Allen, D. E. Interconnection, and A. Beta, 2009, "Tracking the Eastern Interconnection Frequency Governing Characteristic," in 2010 IEEE Power and Energy Society General Meeting, pp. 1-6.

S. Kamboj, W. Kempton, and K. S. Decker, 2011. "Deploying Power Grid-Integrated Electric Vehicles as a Multi-Agent System," 10th Int. Conf. on Autonomous Agents and Multiagent Systems - Innovative Applications Track (AAMAS), vol. 1, no. Aamas, pp. 2-6.

W. Kempton and S. Letendre, 1997. "Electric Vehicles as a new power electric utilities source for electric utilities," Transportation Research Part D: Transport and Environment, vol. 2, no. 3, pp. 157-175.

W. Kempton and J. Tomić, 2005a. "Vehicle-to-grid power fundamentals: Calculating capacity and net revenue,” Journal of Power Sources, vol. 144, no. 1, pp. 268-279.

W. Kempton and J. Tomić, 2005b. "Vehicle-to-grid power implementation: From stabilizing the grid to supporting large-scale renewable energy," Journal of Power Sources, vol. 144, no. 1, pp. 280-294.

National Grid, 2012a. Balancing code 3: Frequency control process.

National Grid, 2012b. CUSC - SECTION 4 BALANCING SERVICES.

National Grid, 2013. Grid Code Chapter 06 - Connection conditions.

PJM, 2012. Manual 12: Balancing Operations.

PJM, 2013a. Manual 1: Control Center and Data Exchange Requirements Control Center Requirements.

PJM, 2013b. Manual 10: Pre-Scheduling Operations,

PJM, 2013c. Manual 11 : Energy \& Ancillary Services

R. Raineri, S. Rios, and D. Schiele, 2006. "Technical and economic aspects of ancillary services markets in the electric power industry : an international comparison," Energy Policy, vol. 34, no. 1010750, pp. 15401555.

V. Rious, J.-M. Glachant, Y. Perez, and P. Dessante, 2008. “The diversity of design of TSOs," Energy Policy, vol. 36, no. 9, pp. 3323-3332. 
RTE, 2004. "Mémento de la sûreté du système électrique".

RTE, 2011a. Documentation Technique de Référence Chapitre 4.1 - Contribution des utilisateurs aux performances du RPT.

RTE, 2011b. Documentation technique de référence Article 8.10 - Modèle de contrat de participation aux services système.

R. Wilson, 2002. “Architecture of Power Markets," Econometrica, vol. 70, no. 4, pp. 1299-1340, [Online]. Available: http://doi.wiley.com/ 10.1111/1468-0262.00334 .

If such a dual payment is implemented, the net metering of energy flows, i.e. the fact that energy flowing from the EV to the grid would be paid the same price as the energy flowing from the grid to the $\mathrm{EV}$, is not required any more. 